\title{
In vivo appearances of gallbladder carcinoma under magnifying endoscopy and probe-based confocal laser endomicroscopy after endosono- graphic gallbladder drainage
}
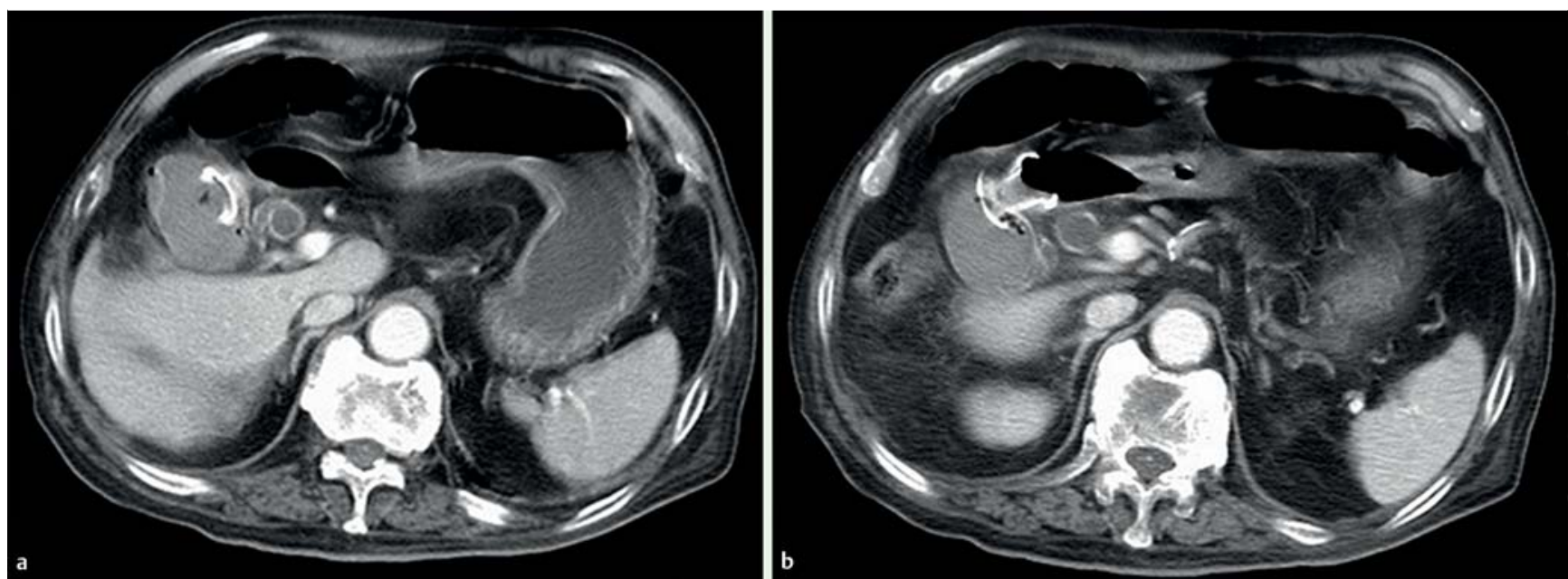

Fig. 1 a, b Computed tomography showing the lumen-apposing stent drainage of the gallbladder to the first part of the duodenum.
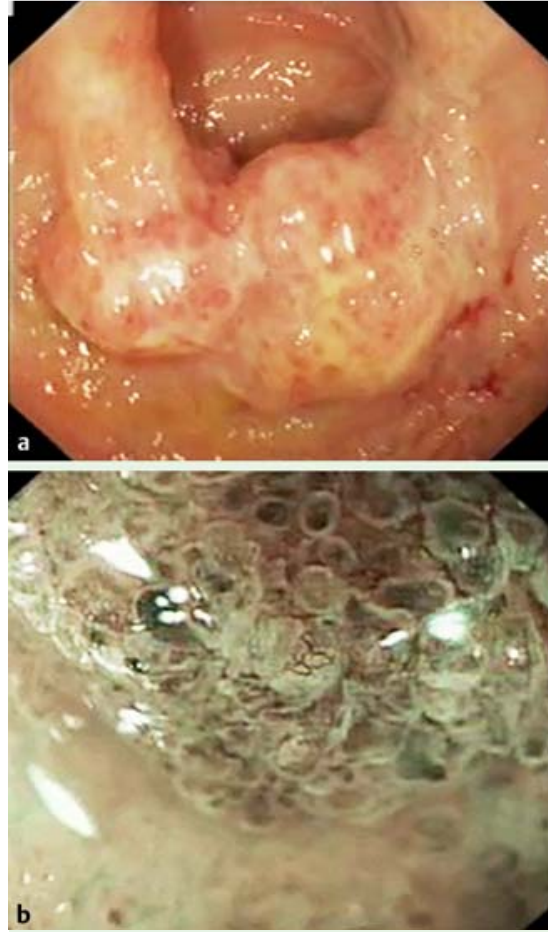

Fig. 2 Gallbladder adenocarcinoma in an 87-year-old man, seen at follow-up endoscopy 3 months after endosonographic gallbladder drainage with a lumen-apposing stent: a white light endoscopic view; $\mathbf{b}$ magnified narrow band imaging (NBI) appearance.
An 87-year-old man with multiple medical co-morbidities was admitted for right upper quadrant pain and fever. Computed tomography showed a distended gallbladder with gallstones and pericholecystic fluid compatible with a diagnosis of acute cholecystitis. There was no gallbladder mass. Since the patient had sepsis and was unfit for surgery, endoscopic ultrasound (EUS) drainage of the gallbladder was performed with a lumen-apposing stent $(10 \times 15 \mathrm{~mm}$, AXIOS; Xlumena, Mountain View, California, USA) as an alternative to percutaneous cholecystostomy ( Fig. 1 a,b). The patient had an uneventful recovery.

Follow-up cholecystoscopy performed 3 months later showed clearance of all stones. However, a 2-cm polypoid lesion was noted at the fundus of the gallbladder. Magnifying narrow band imaging (NBI) showed enlarged and irregular mucosal glands with dilated and corkscrew-appearance microvasculature that was suspicious for malignancy ( Fig. 2). Probe-based confocal laser endomicroscopy (CLE) (GastroFlex; Mauna Kea Technologies, France) showed darkened and irregular columnar cells with loss of villous architecture ( $\mathbf{F i g . 3 a , b )}$. Miniprobe EUS examination (UM-DP12-25R; Olympus, Tokyo, Japan) showed suspicion of tumor involvement of the gallbladder muscularis propria ( Fig.4). Final histological findings confirmed the presence of a gallbladder adenocarcinoma ( $\bullet$ Fig.5). The patient was then treated conservatively as he was too frail to undergo any major surgery.

To our knowledge, this is the first description of a gallbladder adenocarcinoma discovered endoscopically. This was made possible through the use of endosonographic drainage that allowed endoscopic assessment of the gallbladder [1,2]. Magnifying NBI endoscopy has revolutionized the diagnosis of early gastrointestinal neoplasms and is pivotal to performance of endoscopic mucosal resection or submucosal dissection [3]. CLE allows in vivo assessment of cellular architecture and has been shown to be associated with high sensitivity and specificity for diagnosis of Barrett's metaplasia and biliary malignancy [4]. EUS-guided gallbladder drainage in this patient opened the way for application of the above instruments in aiding diagnosis that was not suspected on CT.

Endoscopy_UCTN_Code_CCL_1AZ_2AC

Competing interests: None 

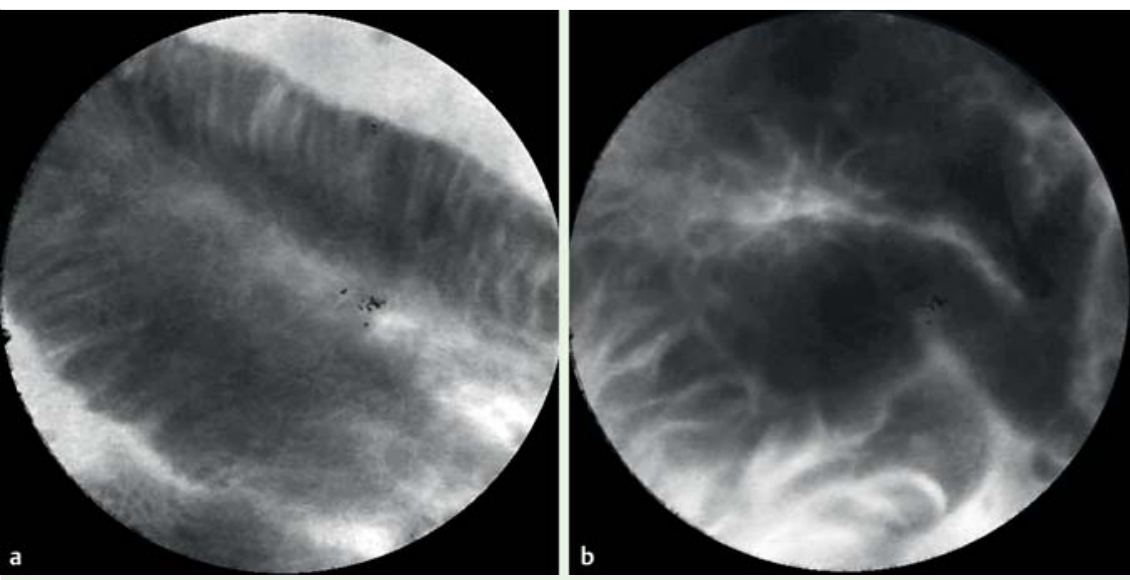

Fig. 3 a Confocal laser endomicroscopy (CLE) image of the normal-looking gallbladder mucosa, showing villous architecture with columnar epithelium. $\mathbf{b}$ CLE image of the gallbladder adenocarcinoma, displaying marked darkened, variably sized glands.

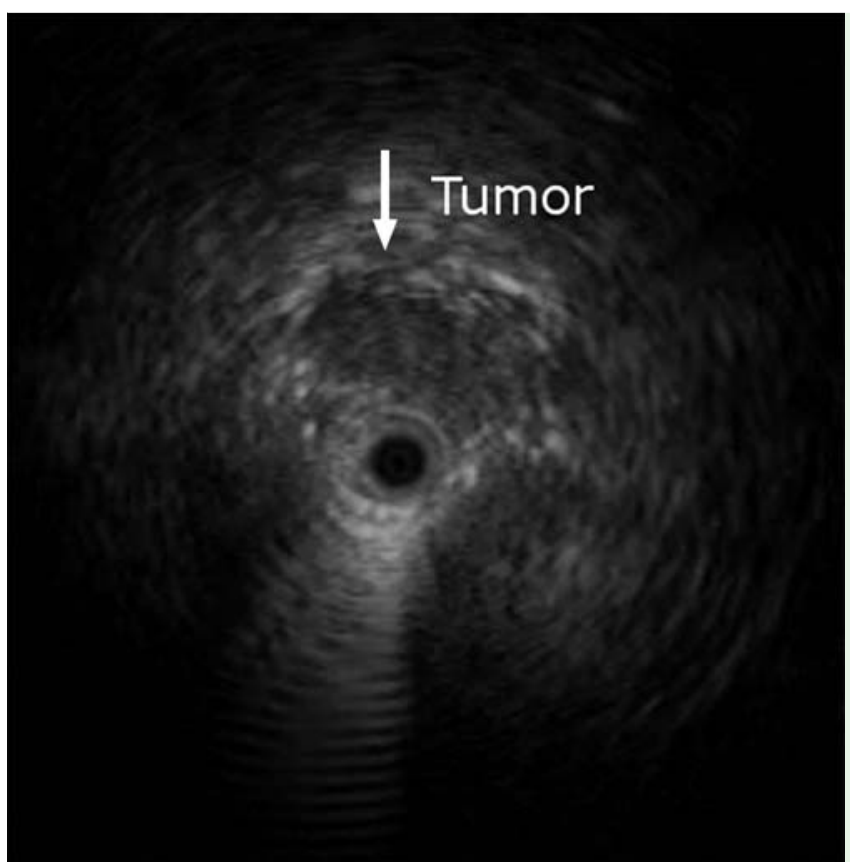

Fig.4 Miniprobe examination with endoscopic ultrasound (EUS) showed tumor involvement of the muscularis propria of the gallbladder.

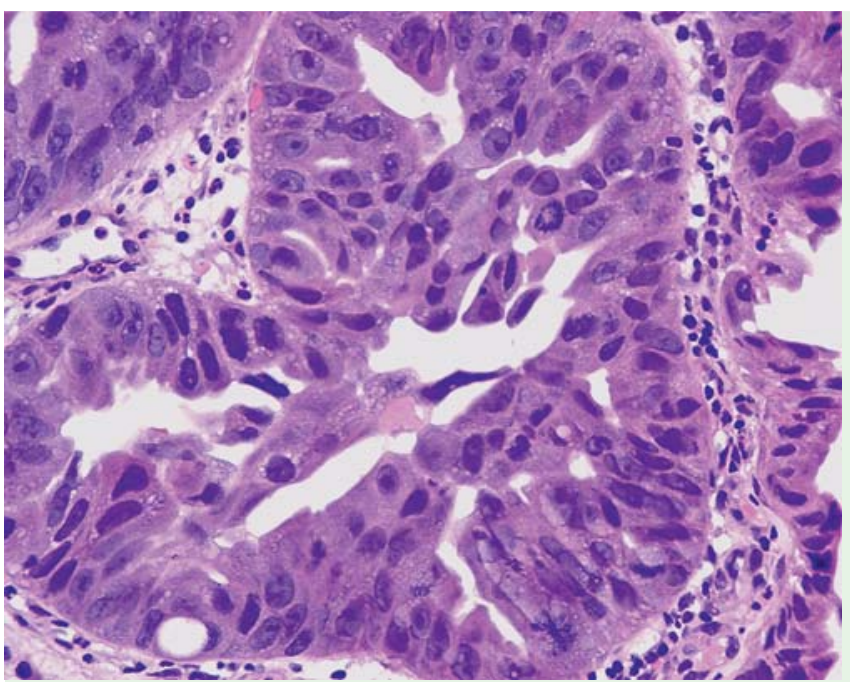

\section{Anthony Y. B. Teoh ${ }^{1}$, Anthony} W. H. Chan' ${ }^{2}$, Philip W. Y. Chiu', James Y. W. Lau' ${ }^{1}$

${ }^{1}$ Department of Surgery, Prince of Wales Hospital, The Chinese University of Hong Kong, Hong Kong SAR

2 Department of Anatomical and Cellular Pathology, Prince of Wales Hospital, The Chinese University of Hong Kong, Hong Kong SAR

\section{References}

1 de la Serna-Higuera C, Pérez-Miranda M, GilSimón $P$ et al. EUS-guided transenteric gallbladder drainage with a new fistula-forming, lumen-apposing metal stent. Gastrointest Endosc 2013; 77: 303-308

2 Itoi T, Binmoeller KF, Shah J et al. Clinical evaluation of a novel lumen-apposing metal stent for endosonography-guided pancreatic pseudocyst and gallbladder drainage (with videos). Gastrointest Endosc 2012; 75: $870-876$

3 Endoscopic Classification Review Group. Update on the Paris Classification of superficial neoplastic lesions in the digestive tract. Endoscopy 2005; 37: 570-578

4 Nakai Y, Isayama H, Shinoura S et al. Confocal laser endomicroscopy in gastrointestinal and pancreatobiliary diseases. Dig Endosc 28. 08. 2013. doi: 10.1111/den.12152 [Epub ahead of print]

\section{Bibliography}

Dol http://dx.doi.org/

10.1055/s-0033-1359139

Endoscopy 2014; 46: E13-E14

(c) Georg Thieme Verlag KG

Stuttgart · New York

ISSN 0013-726X

\section{Corresponding author}

\section{Anthony Y. B. Teoh, MD}

Department of Surgery, Prince of Wales Hospital

The Chinese University of Hong Kong

Shatin, Hong Kong

Fax: +852-2637-7974

anthonyteoh@surgery.cuhk.edu.hk 